\title{
Predicción Temporal de la Epidemia de Dengue en Colombia: Dinámica Probabilista de la Epidemia
}

\section{Temporal prediction of the dengue epidemic in Colombia: the epidemic's probabilistic dynamics}

\author{
Javier Rodríguez y Catalina Correa \\ Centro de Investigaciones, Clínica del Country. grupoinsight@yahoo.es, scatalinacorreah@hotmail.com \\ Recibido 24 Septiembre 2008/Enviado para Modificación 8 Abril 2009/Aceptado 15 Mayo 2009
}

\section{RESUMEN}

Objetivo Estudiar la dinámica geométrica del número de casos anuales de Dengue registrados en Colombia durante los años 1990-2006 haciendo una analogía con una caminata al azar probabilista.

Métodos Se analizó matemáticamente la dinámica de la epidemia de Dengue en Colombia desde 1990 hasta el 2006 como una caminata al azar probabilista, construyendo el espacio total de probabilidades para la dinámica para analizar el comportamiento probabilista de aumentos y disminuciones consecutivos y el comportamiento probabilista de casos durante rangos de años consecutivos, calculando la predicción temporal de los casos anual.

Resultados El rango de valores predicho para el número de infectados en el año 2007 varió entre 57581 y 20008 , resultado que fue refinado con el estudio probabilista de las variaciones anuales encontrando un valor de 48188 para el número de infectados en el año 2007. Posteriormente esta predicción fue corroborada con los datos del Instituto Nacional de Salud de Colombia con un valor real de 43564 infectados registrados al 31 de diciembre, valor que corresponde al 90,4\% respecto al valor predicho.

Conclusión Se encontró un orden matemático acausal de la epidemia de Dengue en Colombia que permite realizar predicciones temporales, simples y útiles en las decisiones de salud pública.

Palabras Clave: Predicción, probabilidad, Dengue, (fuente: DeCS, BIREME).

\section{ABSTRACT}

Objective Studying geometric dynamics for annual cases of dengue recorded in Colombia from 1990-2006 by making an analogy with a probabilistic random walk.

Methods The geometric dynamics for the number of annual cases of dengue registered in Colombia for 1990-2006 was mathematically analyzed as a probabilistic random walk, building the total probability space for the dynamics in order to analyze the probabilistic behavior of consecutive increases and decreases and the probabilistic behavior of cases during ranges of serial years and thus calculate the temporary prediction of annual cases. 
Results The extreme values predicted for the number of infected people during 2007 were 57581 and 20 008; the predicted value was refined by analysing annual variations, giving a 48188 predicted value for the number of infected people in 2007 . This prediction was then corroborated by using Colombian National Institute of Health data having a 43564 recorded real infected value on December $31^{\text {st; }}$; the real value corresponded to $90,4 \%$ regarding predicted value.

Conclusion A mathematical and acausal order for the dengue epidemic in Colombia was found from the probabilistic random walk; thus led to carrying out temporary, simple and useful predictions in the public health decision-making.

Key Words: Probability, forecasting, Dengue (source: MeSH, NLM).

a fiebre del Dengue es una enfermedad viral aguda febril, que se transmite al hombre a través de la picadura de mosquito del género Aedes aegyptiy y la mayoría de los infectados desarrollan la forma leve o dengue clásico (DC) y algunos desarrollan el tipo hemorrágico (DH) con menor frecuencia (1). La Prevalencia Global del dengue ha aumentado de forma dramática en las últimas décadas. Esta enfermedad se considera endémica en más de 100 países de África, el Este Mediterráneo, el sureste de Asia y el Oeste del Pacífico. Cerca de 2500 millones de personas tienen riesgo de contraer Dengue. La Organización Mundial de la Salud estimó hacia el año 2002 que cerca de 50 millones de casos de dengue ocurrían a nivel mundial anualmente (2).

Se ha documentado en los últimos 20 años un aumento en América de un total de 492820 casos, de los cuales 77000 corresponden a DH $(3,4)$. Para el año 2007 hasta la semana epidemiológica 39 el número de casos de Dengue en las Américas estuvo alrededor de 630356 (5). En Colombia el número de casos ha aumentado en los últimos 10 años, y para la semana epidemiológica 50 del 2007 se reportaron 41773 casos por la Organización Panamericana de la Salud $(3,5)$.

La cuantificación de la posibilidad de que un evento ocurra es posible a través de la probabilidad, la cual es evaluada a partir de la construcción del espacio muestral, el cual contiene los valores matemáticos adimensionales de todos los posibles resultados de un experimento (6-9). La geometría fractal ha caracterizado el movimiento Browniano (10), el cual ha permitido descubrimientos científicos de gran relevancia; la deducción incontrovertible de la existencia del átomo (11), y ha sido aplicado en varios modelos teóricos y económicos (12-14). En este trabajo se aplicó el modelo desarrollado por Wiener (15), el cual corresponde a un modelo teórico probabilista con representaciones de caminatas aleatorias (7). Dado que la epidemia de dengue en el tiempo es un fenómeno 
irregular y complejo, se estudiará a partir del movimiento browniano, pues este presenta características similares en el tiempo.

Por medio de la caminata al azar que consiste en la asociación de una probabilidad a un desplazamiento en una dirección determinada, por ejemplo la asignación de un desplazamiento hacia arriba para la probabilidad de cara y hacia abajo para la probabilidad de sello, de tal manera que con la secuencia de probabilidades se va construyendo la caminata, se puede representar y caracterizar un movimiento probabilista acotando su dinámica, encontrando así valores numéricos que permitan predecir dentro de ciertos rangos la evolución de una dinámica (7). En este trabajo se utilizó la caminata al azar para caracterizar la evolución dinámica anual de la epidemia de Dengue. Una de las medidas de la caminata al azar es la desviación media cuadrática, la cual permite cuantificar si los eventos específicos del espacio muestral presentan un cargamiento de la probabilidad hacia valores específicos; comparándola con su valor esperado (7).

La mayor parte de los estudios de la epidemia se han ocupado del análisis espacio-temporal de este fenómeno (16-18), desarrollando modelos matemáticos a partir de registros del número de infectados en zonas geográficas específicas (19) y modelos descriptivos basados en el clima (20,21), que se orientan a la comprensión de los múltiples factores que participan en la dinámica de la epidemia del Dengue, evidenciando dificultades en la predicción. Dentro de los macrofactores más importantes que coinciden con el incremento del número de casos de Dengue, son los cambios en el clima, el crecimiento, y movimiento poblacional (5). Este trabajo plantea una nueva visión para el análisis de este fenómeno realizando una abstracción de todos estos macrofactores, y así estudiarlo a partir de las teorías físicas y matemáticas para buscar un orden acausal subyacente. En la mecánica newtoniana la causa del movimiento son las fuerzas, la ley de fuerza. Desde la mecánica estadística, la mecánica cuántica y teoría del caos no hay causas del movimiento sino un orden matemático que describe y predice los fenómenos. Por ejemplo en la teoría cuántica se predice probabilistamente el movimiento del electrón, no qué lo causa. En este sentido es usada la concepción acausal aquí.

En un trabajo realizado por Rodríguez y Bernal, se realizó una predicción temporal de la epidemia de Malaria en Colombia para el año 2007; que corresponde al 95,6 \% respecto al número de casos reportados por el Instituto Nacional de Salud de Colombia (INS). En este trabajo se realiza una aplicación de la misma metodología para la predicción del número de infectados de Dengue para el año 2007. 
Dado que la epidemia de dengue en el tiempo es un fenómeno irregular y complejo, se utilizará la caminata al azar probabilista y la teoría de la probabilidad con el objetivo de estudiar de forma objetiva y reproducible su dinámica y de esta forma caracterizar su evolución y realizar predicciones para el número de casos de Dengue en el tiempo.

\section{METODOLOGÍA}

Definiciones

Longitud: La longitud L de una variación anual se define como:

$$
L=\sqrt{\left(X_{0}-X_{0}\right)^{2}+\left(Y_{0}-Y_{1}\right)^{2}} \quad \text { Ecuación } 1
$$

Donde $\mathrm{X}_{0}$ y $\mathrm{Y}_{0}$ son las coordenadas cartesianas del valor correspondiente al año inicial y $\mathrm{X}_{0}$ y $\mathrm{Y}_{1}$ son las coordenadas cartesianas del año siguiente, debido al modelo de caminata al azar usado en este estudio, el valor de las coordenadas en $\mathrm{X}$ son las mismas pues el movimiento es estudiado solamente en el eje de las Y, esto es, la variación en el eje $\mathrm{X}$ para cada año es cero, y los valores para $\mathrm{Y}$ corresponden al número de infectados reportados para cada año.

Probabilidad de una longitud: La probabilidad de la longitud L de una variación anual se define como la longitud de dicha variación dividida entre el total de las longitudes (7):

$$
P(L)=\frac{\text { Longitud } \text { variación anual }}{\text { Total longitudes }}=\frac{L}{T L} \quad \text { Ecuación } 2
$$

Probabilidad de casos infectados: La probabilidad del número de casos de dengue reportados en un año se define como el número de casos para dicho año dividido entre el total de casos (7):

$$
P(N)=\frac{\text { Número casos anual }}{\text { Total casos }} \quad \text { Ecuación } 3
$$

Desviación media cuadrática del número de casos: Para estudiar si los valores del número de casos son equiprobables o no, es decir si hay un cargamiento 
de la probabilidad para eventos específicos del espacio muestral, usamos la fórmula de la desviación equiprobable de la caminata al azar (7):

$P(R n)=\frac{\text { Número casos anual }}{\text { Total casos }} \pm \frac{1}{2 \sqrt{N}} \quad$ Ecuación 4

Donde $\mathrm{N}$ es el número de eventos del espacio muestral, esto es el total de casos.

Inicialmente se construyó una grafica con el número de casos de infectados de Dengue, y se calcularon las distancias de todas las variaciones anuales, aplicando la ecuación 1 y construyendo el espacio total de probabilidad de la dinámica de la epidemia, donde cada variación anual se define como un evento. Luego se calcularon los valores de probabilidad para cada longitud correspondiente a cada variación anual aplicando la ecuación 2, para calcular después la proporción de cada variación anual respecto a la distancia mínima encontrada.

Se construyó un espacio de probabilidad para el período 1990-2006 que cuantifica el número de infectados anuales, y se calcularon los valores de probabilidad de infección anual aplicando la ecuación 3, para calcular los valores de la desviación media cuadrática aplicando la ecuación 4 , y después calcular las diferencias entre el valor esperado y su desviación; para estudiar si las probabilidades en este rango son equiprobables o no lo son. Este mismo procedimiento se realizó para dos espacios de probabilidad cuantificados para dos periodos de tiempo19901997 y 1998-2006. Estos dos rangos temporales se escogieron porque presentan valores en el número de infectados bien diferenciados en la dinámica de la epidemia.

Se definió un nuevo espacio de probabilidad que solamente posee dos eventos: disminución y aumento del número de casos con respecto al año anterior, para estudiar la dinámica de la dinámica como si fueran los dos eventos del lanzamiento de una moneda, en períodos consecutivos de dos y tres años, para el período 1990-2006; partiendo de estos cálculos se selecciona el valor cuantitativo para la predicción del año 2007 que corresponda a la combinación respecto a los dos años anteriores y con mayor probabilidad en las variaciones consecutivas de tres años.

Posteriormente se construyó un espacio de probabilidad que evalúa los últimos tres años consecutivos, con el fin de delimitar la dinámica de la epidemia y 
determinar la predicción; definiendo primero, el promedio aritmético de estos tres años como el valor de la probabilidad para la longitud del año 2007; segundo, se reemplazó este valor en la ecuación 2 y se desarrolló hasta obtener una ecuación cuadrática en función de $\mathrm{Y}_{1}$, ecuación 5 , obteniendo como solución dos valores que corresponden a la predicción para el año 2007.

$$
Y_{2007}=\frac{2 Y_{2006} \pm \sqrt{\left(-2 Y_{2006}\right)^{2}-4\left\{Y_{2006}{ }^{2}+\left(X_{0}-X_{0}\right)^{2}-\left[\left(P_{(L)}\right)^{2} \times(T L)^{2}\right]\right\}}}{2}
$$

\section{Ecuación 5}

Donde: $\mathrm{P}_{(\mathrm{L})}$ Es el promedio aritmético de la probabilidad para los tres últimos años, y TL es la sumatoria de las distancias para este mismo rango. En este punto es fundamental abstraer los datos del número de casos como una caminata al azar, para convertirla en probabilidades y partiendo de éstas realizar nuevamente uno de los segmentos de la caminata predictiva, el cual corresponde al año 2007.

\section{RESULTADOS}

Los valores para las distancias para los casos anuales de infectados, variaron entre 1 955- 41 625, siendo el valor mínimo de este rango el correspondiente al Patrón de Comparación, los datos no se muestran. Los valores para las proporciones respecto al patrón para los casos anuales de infectados, se encontraron en rangos de 1-21,3, los datos no se muestran. Los valores de probabilidad de la longitud de la variación para los casos anuales de infectados, se encontraron en rangos de 0,008-0,17.

Los valores de probabilidad para los casos anuales reportados en el período 1990-2006 variaron entre 0,3 y 0,1, los valores de la desviación media cuadrática para estos valores variaron en un rango de 0,02 a 0,1 , encontrando que las diferencias entre estos últimos y el valor esperado varían en un rango de -0,0006 a 0,0006, los datos no se muestran. Los valores de probabilidad para el número de casos anuales reportados que se cuantificaron en los dos períodos 1990-1997 y 1998-2006 variaron en un rango de 0,05 a 0,2, los valores de la desviación media cuadrática para estos valores variaron en un rango de 0,05 a 0,2 , encontrando que las diferencias entre estos últimos y el valor esperado varían en un rango de $-0,0008$ a 0,001 (Tabla 1). Los diferencias entre los valores de la desviación media cuadrática muestran cómo el comportamiento de la epidemia se encuen- 
tra más cargado en todo el período de 1990-2006 que en periodos divididos, evidenciando que los rangos de acotamiento del número de casos es más determinista y equiprobable entre el período 1990-2006.

Tabla 1. Valores de probabilidad $(P)$, desviación media cuadrática (+Dmc, -Dmc) y diferencia entre estos, (+Dmc-P),(-Dmc-P), para los períodos 1990-1997, 1998-2006

\begin{tabular}{cccccc}
\hline Año & $P$ & +Dmc & -Dmc & +Dmc -P & -Dmc -P \\
\hline 1990 & 0,09 & 0,1 & 0,1 & 0,001 & $-0,001$ \\
1991 & 0,07 & 0,1 & 0,1 & 0,001 & $-0,001$ \\
1992 & 0,1 & 0,1 & 0,1 & 0,001 & $-0,001$ \\
1993 & 0,1 & 0,1 & 0,1 & 0,001 & $-0,001$ \\
1994 & 0,1 & 0,1 & 0,1 & 0,001 & $-0,001$ \\
1995 & 0,1 & 0,1 & 0,1 & 0,001 & $-0,001$ \\
1996 & 0,2 & 0,2 & 0,2 & 0,001 & $-0,001$ \\
1997 & 0,2 & 0,2 & 0,2 & 0,001 & $-0,001$ \\
1998 & 0,2 & 0,2 & 0,2 & 0,0008 & $-0,0008$ \\
1999 & 0,1 & 0,1 & 0,1 & 0,0008 & $-0,0008$ \\
2000 & 0,1 & 0,1 & 0,1 & 0,0008 & $-0,0008$ \\
2001 & 0,1 & 0,1 & 0,1 & 0,0008 & $-0,0008$ \\
2002 & 0,2 & 0,2 & 0,2 & 0,0008 & $-0,0008$ \\
2003 & 0,1 & 0,1 & 0,1 & 0,0008 & $-0,0008$ \\
2004 & 0,1 & 0,1 & 0,1 & 0,0008 & $-0,0008$ \\
2005 & 0,1 & 0,1 & 0,1 & 0,0008 & $-0,0008$ \\
2006 & 0,1 & 0,1 & 0,1 & 0,0008 & $-0,0008$ \\
\hline
\end{tabular}

Tabla 2. Frecuencia y probabilidad para aumentos y disminuciones consecutivos en el período 1990-2006, y valor total de cada tipo de variación

\begin{tabular}{ccccc}
\hline $\begin{array}{c}\text { Número años } \\
\text { consecutivos } \\
(1990-2006)\end{array}$ & \multicolumn{2}{c}{ Aumentos } & \multicolumn{2}{c}{ Disminuciones } \\
\hline 1 & 1 & 0,06 & 4 & 0.25 \\
2 & 0 & 0 & 1 & 0,13 \\
3 & 3 & 0,56 & 0 & 0 \\
Total & 10 & 0,62 & 6 & 0,38 \\
\hline
\end{tabular}

Respecto a la cuantificación de aumentos y disminuciones para dos años consecutivos se encontró que para el período de 1990 a 2006, el número de aumentos consecutivos varió entre uno y tres años y el número de disminuciones consecutivas entre uno y dos años, encontrando que aumentos de dos años, así como disminuciones mayores de dos años consecutivos tienen un valor de cero (Tabla 2). Se encontró que el número de posibles combinaciones de aumentos y disminuciones para un período de tres años es igual a 8, (Tabla 3). Encontrando 
que para el período final de tres años (2005-2007), dado que el 2005 aumentó y el 2006 disminuyó, la probabilidad de que el año 2007 aumente es el doble de la probabilidad de que disminuya (Tabla 3). El valor promedio de la probabilidad de la variación anual para el período 2004-2006 es 0,3, encontrándose que los dos valores predichos del número de casos para el 2007 fueron 57582 y 20008 .

Dado que la probabilidad de que el año 2007 aumente con respecto al año anterior es el doble de la probabilidad de que disminuya, se predice que el valor para el año 2007 aumentará respecto al año anterior, y debido a que existe una probabilidad de que disminuya, el valor predicho para el año 2007 corresponde al valor medio entre el valor promedio de los dos valores predichos del número de casos para el 2007 y el límite superior, esto es 48188 infectados (Figura 1), el cual fue corroborado con los datos reportados por el INS en la semana 52, donde se habían reportado 43564 infectados, encontrando que el valor real corresponde al 90,4\% respecto al valor predicho, este valor no corresponde al consolidado final, sino al acumulado para la fecha mencionada.

Tabla 3. Combinaciones posibles de aumentos $(A)$ y disminuciones (D) para un período de tres años consecutivos

\begin{tabular}{ccc}
\hline Combinaciones & Valor & Probabilidad \\
\hline AAA & 3 & 0,2 \\
DDD & 0 & 0 \\
AAD & 3 & 0,2 \\
DDA & 1 & 0,07 \\
ADD & 1 & 0,07 \\
DAA & 3 & 0,2 \\
ADA & 2 & 0,1 \\
DAD & 1 & 0,07 \\
Total & 14 & 1 \\
\hline
\end{tabular}

\section{DISCUSIÓN}

Este es el primer trabajo en el que basados en una longitud probabilista para construir una caminata probabilista, se predice la dinámica temporal de la epidemia de dengue en Colombia, de forma práctica y útil en la toma de decisiones en la salud pública. 
Figura 1. Valor predicho para el año 2007, y valor real del acumulado reportado por el INS correspondiente al día 22 de diciembre de 2007

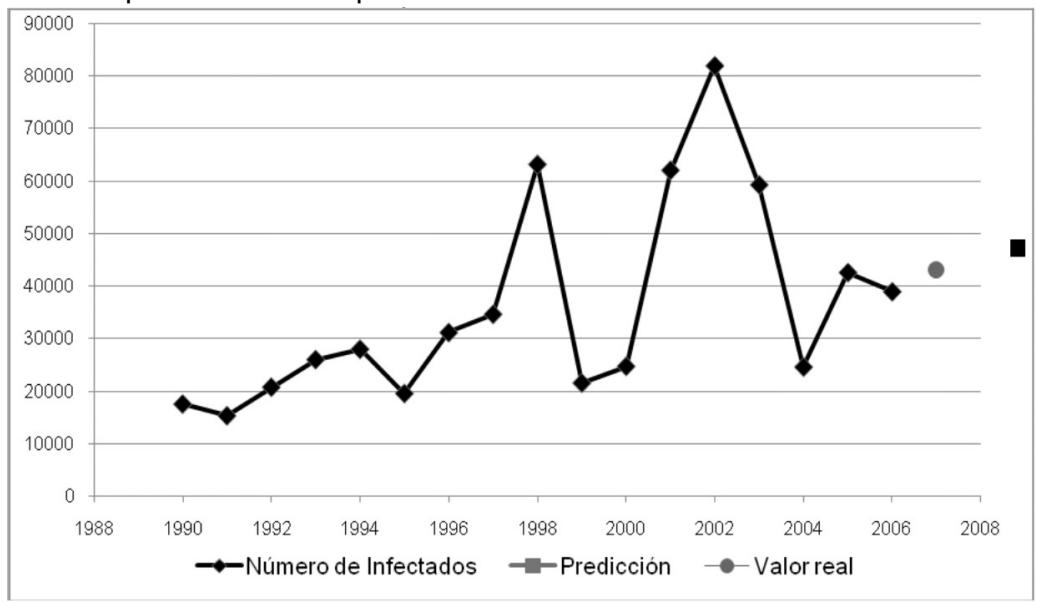

Convertir la cantidad de infectados en cada año consecutivo en longitudes probabilistas y con la variación de los últimos años consecutivos para predecir el número de casos para el 2007 mostró cómo la analogía con una caminata al azar resulta útil y práctica al tener posibles rangos, de acuerdo con la realidad de la dinámica de la epidemia. Para que se pueda aplicar de forma sistemática esta metodología a una dinámica debe cumplir con la función de la recta, los axiomas de la probabilidad y tener variaciones temporales constantes, para así poder realizar una asociación entre las rectas y los valores de probabilidad realizando una predicción dinámica probabilista, es decir, una nueva recta; esta sistematización también es aplicable a cualquier fenómeno que presente movimiento Browniano, pues es una generalización de éste. La probabilidad para las longitudes anuales se diferencia de la probabilidad para el número de casos anuales en que la primera cuantifica variaciones geométricas año a año, mientras que la segunda cuantifica el número total de casos anuales respecto a la totalidad, adicionalmente la primera fue utilizada para realizar la predicción en el contexto de la solución de las ecuaciones de segundo grado, y la segunda se utilizó para estudiar el cargamiento de la probabilidad de la dinámica de la epidemia.

En este trabajo, se realizó una abstracción del tiempo al estudiar las variaciones sólo en un eje, lo que permitió una simplificación de la distancia pitagórica para la realización de predicciones en el tiempo. Los modelos dinámicos consideran múltiples causas, el clima, vectores; esta metodología, a partir del pensamiento acausal, simplifica esta forma de ver el fenómeno, abstrayendo las causas, para desde una dinámica probabilista sin causas realizar predicciones sobre el 
fenómeno. Esta predicción es apropiada porque la dinámica de la epidemia está cargada en la región estudiada lo que es evidenciado con las diferencias entre las desviaciones cuadráticas, hallazgo fundamental y práctico para entender el fenómeno y así poder desarrollar la predicción en el tiempo.

Con los aumentos y disminuciones consecutivas y los cargamientos de probabilidades se desarrolla una predicción determinista en el contexto acausal probabilista lo que sorprende frente a los supuestos prejuicios de aleatoriedad o caos. Los estudios matemáticos con múltiples interacciones de factores y análisis espacio-temporales son dependientes de las interacciones que se consideran causantes de la infección (16-21), a diferencia de este trabajo físico-matemático acausal y predictivo.

La dinámica de la epidemia refleja un comportamiento probabilista pues la probabilidad de que aumente es el doble, 0,14 , que la probabilidad de que disminuya, 0,07 , en los cálculos de todas las posibles combinaciones de aumentos y disminuciones para tres años consecutivos, lo que da un sustento mas fuerte a las relaciones predictivas consecutivas, y predice una mayor probabilidad de aumento para el año 2007 escogiendo así el valor intermedio entre el valor medio de los valores predichos y el valor superior.

Para corroborar el acierto en la predicción se contrastó el número real de casos reportados por el INS con el valor de la predicción, tomando el valor predicho como el porcentaje total sobre el cual se realiza la comparación con el valor real. En este trabajo se encontró que aunque los valores para el número de casos registrados cambian en el tiempo, los valores límites se mantienen más o menos constantes en períodos de tiempo consecutivos; lo cual denota que existe una "inercia probabilista" en la dinámica de la epidemia, la cual es cuantificada con la desviación media cuadrática para los valores de probabilidad en el período total

Agradecimientos. A Signed Prieto y Pedro Bernal por la ayuda en la elaboración de este manuscrito y por la discusión de los conceptos y las metodologías. A Salua Osorio, Viviana Cerón y Patricia Gutiérrez, del INS por apoyar la investigación y facilitarnos los datos para realizar este trabajo. A la Dra. Laura Pinilla y a la fisioterapeuta Diana Cardona por su colaboración en la redacción y organización del artículo. 


\section{REFERENCIAS}

1. World Health Organization. Chapter 1: General Considerations. In: Dengue haemorrhagic fever: diagnosis, treatment, prevention and control. 2nd edition. Geneva: World Health Organization; 1997. p.1-11.

2. Dengue and dengue haemorrhagic fever. Fact sheet $N^{\circ} 117$. March 2009. [Internet]. Disponible en: http://www.who.int/mediacentre/factsheets/fs117/en Consultado Abril del 2009.

3. Ocazionez RE, Gómez SY, Cortés FM. Serotipo, Patrón de Infección y Dengue Hemorrágico en Área Endémica Colombiana. Rev. Salud Pública 2007; 9(2): 262-274.

4. Guzmán MG, Kouri G. Dengue and dengue hemorrhagic in the Americas: lessons and challenges J Clin Virol 2003; 27(1):1-3

5. EER Noticias: Enfermedades infecciosas emergentes y reemergentes, Región de las Américas. 26 septiembre 2007; 4(9) [Internet]. Disponible en: http://www.paho.org/spanish/ad/ dpc/cd/eid-eer-2007-09-26.htm. Consultado Abril del 2009.

6. Laplace P. Ensayo filósofico sobre las probabilidades. Barcelona: Altaza; 1995.

7. Feynman RP, Leighton RB, Sands M. Probabilidad. En: Feynman RP, Leighton RB, Sands M. Física. Vol. 1. Wilmington: Addison-Wesley lberoamericana, S. A. 1964; p. 6-1, 6-16.

8. Mood A, Graybill F, Boes D. Introduction to the theory of statistics. 3a Ed. Singapore: Mc. GrawHill; 1974.

9. Blanco L. Probabilidad, notas de clase. Bogotá: Universidad Nacional de Colombia. Departamento de Matemáticas y Estadística; 1996.

10. Mandelbrot B. Los Objetos Fractales. Barcelona: Tusquets Eds. S.A.; 2000.

11. Einstein $A$. Sobre el movimiento de partículas pequeñas suspendidas en líquidos en reposo exigido por la teoría cinético-molecular del calor. En: Stachel J, ed. Einstein 1905 un año milagroso: cinco artículos que cambiaron la física. Barcelona: Crítica; 2001.

12. Saupe D. Simulation of Brownian Motion, Irregular Shapes: Randomness in Fractal Constructions. En Peitgen H, Jurgens H, Saupe D. Chaos and Fractals: New Frontiers of Science. N.Y.: Springer-Verlag; 1992.

13. Mandelbrot B, Van Ness JW. Fractional Brownian Motions, fractional Brownian noises and applications. SIAM Review 1968; 10(4): 422-37.

14. Mandelbrot, B. The behavior of certain speculative prices. The Journal of Business 1963; 36: 394-419.

15. Wiener N. Nonlinear problems in random theory. Cambridge: Technology Press; 1958.

16. Rotela C, Fouque F, Lamfri M, Sabatier P, Introini V, Zaidenberg M, Scavuzzo C. Space-time analysis of the dengue spreading dynamics in the 2004 Tartagal outbreak, Northern Argentina. Acta Trop 2007; 103(1):1-13.

17. Morrison AC, Getis A, Santiago M, Rigau-Perez JG, Reiter P. Exploratory space-time analysis of reported dengue cases during an outbreak in Florida, Puerto Rico, 1991-1992. Am J Trop Med Hyg. 1998; 58(3):287-98.

18. Tran A, Deparis X, Dusssart P, Morvan J, Rabarison P, Remy F, et al. Dengue spatial and temporal patterns, French Guiana, 2001. Emerg Infect Dis [serial online] 2004 Apr [date cited]. [Internet]. Disponible en: http://www.cdc.gov/ncidod/EID/vol10no4/03-0186.htm . Consultado Abril del 2009.

19. Chowell G, Diaz-Dueñas P, Miller JC, Alcazar-Velazco A, Hyman JM, Fenimore PW, CastilloChavez C. Estimation of the reproduction number of dengue fever from spatial epidemic data. Math Biosci. 2007; 208(2):571-89.

20. Gerardo Chowell, Fabio Sanchez. Climate-based descriptive models of dengue fever: the 2002 epidemic in Colima, Mexico. J Environ Health 2006; 68(10): 40-44,55.

21. Patz JA, Martens WJ, Focks DA, Jetten TH. Dengue fever epidemic potential as projected by general circulation models of global climate change. Environ Health Perspect 1998; 106(3):147-53. 